\title{
Therapeutic Approach for the Treatment of Three Dermatoses in a Young Patient: A Case Report
}

\author{
Faizullina $\mathrm{E}^{1}$, Faizullin $\mathrm{I}^{2 *}$ and Bodrova $\mathbf{R}^{2}$ \\ ${ }^{1}$ Department of Dermatology, Venereology and STD, Kazan State Medical University, \\ Russian Federation \\ ${ }^{2}$ Department of Rehabilitation and Sports Medicine, Kazan State Medical Academy \\ (KSMA)- Branch Campus of the FSBEIFPE RMACPE, Russian Federation
}

Case Report

Volume 4 Issue 4

Received Date: November 11, 2019

Published Date: December 11, 2019

DOI: $10.23880 /$ cdoaj-16000195

*Corresponding author: Iaroslav Faizullin, Attending physician at the Department of Rehabilitation and Sports Medicine, Kazan State Medical Academy (KSMA)- Branch Campus of the FSBEIFPE RMACPE, Kazan, Russian Federation, Tel: +7(843)236-08-75; Email: iaroslav.faizullin@gmail.com

\section{Abstract}

A 22 year old male patient with a combination of three dermatoses: perioral dermatitis, atopic dermatitis and acne vulgaris constitutes an interesting clinical case, which we would like to present in this article. Having observed the patient for more than two years, we gained a set of data, noteworthy for the field of dermatology. In case of perioral dermatitis, the use of cosmetics and detergents, toothpaste containing fluoride, ointments containing glucocorticoids should be avoided. Topical corticosteroids have proven effective against atopic dermatitis. Should topical corticosteroids, such as hydrocortisone fail to take effect, a short-term treatment with topical calcineurin inhibitors like tacrolimus or pimecrolimus might be considered. In the case at hand, a treatment with retinoids proved to be remedy of choice against acne vulgaris.

Keywords: Acne vulgaris; Atopic dermatitis; Perioral dermatitis

Research Aim: Clinical case analysis of a young patient with a combination of three dermatoses: perioral and atopic dermatitis, acne vulgaris.

\section{Research Objectives}

1. Study of the mechanisms of xerosis formation in patients with combined dermatoses.

2. The formation of treatment approach in a young patient with a combination of three dermatoses.

\section{Case Report}

We report about a 22 y.o male patient who visited a dermatologist for the first time due to the rash dryness of the face. He associated illness with the use of a shaving gel. The patient consulted various medical professionals in Kazan (Russia) to no effect.

St.localis. On the facial skin in the area of the cheeks, the chin area showed a spot-shaped red rash 0.5 to $1.5 \mathrm{~cm}$ in 


\section{Clinical Dermatology Open Access Journal}

diameter, partially scaly, clearly demarcated. Lymph nodes were not enlarged.

Diagnosis: allergic contact dermatitis caused by cosmetic products.

\subsubsection{6}

The patient suffered from a rash and a fever of $38,5^{\circ} \mathrm{C}$ (axillary measurement) for 2 days. He attributed the disease to previous contact with a person suffering from an acute respiratory viral disease.

St.localis. Pathological process localized on the skin of the hands and feet - pink papules, pustules. Lymph nodes were not enlarged.

Diagnosis: Enterovirus infection. A consultation of infectiologist is recommended.

\subsubsection{6}

The patient visited a medical facility because of the rash on his face, he could not identify a cause, no seasonality.

St.localis. Pathological process was limited, asymmetric, located on the facial skin. Erythema and dandruff appeared on the skin of the nasolabial angle. Lymph nodes were not enlarged.

Diagnosis: Seborrheic dermatitis. Allergic dermatitis. Rosacea.

Medication: 1) Cetirisin $10 \mathrm{mg} 1$ tabl. for 7 days.

\subsubsection{7}

The patient visited a dermatologist for control without improvement in condition.

Clinically blue papules showed on the facial skin. Perioral showed dandruff and redness. Peripheral lymph nodes were not enlarged.

Diagnosis: Rosacea. Perioral dermatitis.

Medication: 1) Metronidazole 250mg 1 tabl. X 2 times a day after a meal for 10 days, after that

2) Ornidazole $500 \mathrm{mg} 1$ tabl. $X 2$ times a day for 10 days

3) Vitamins D $15000 \mathrm{IU} / \mathrm{ml} 10$ drops per day, 1 month.

The patient was examined by the specialist in allergology.

There was no evidence of an allergy.

\subsubsection{7}

The patient complained of rash on facial skin for 1 year. The patient was treated several times with various ointments and creams, including hormonals and antifungals in Kazan. Diagnosis: 1) Rosacea. 2) Seborrheic dermatitis 3) Perioral iatrogenic dermatitis.

Medication: 1) 2\% boric acid as a water pad - lotion (boric acid, pharmacological group - antiseptic), as a spray several times a day.

2) zinc ointment (zinc oxide 10\%) 2-3 times a day.

Repeat in 2 weeks.

\subsubsection{7}

There was a positive development: infiltration reduction, mild dandruff. However, it exhibited a slight skin tension paraorbital.

In addition to the treatment: 1) Prednisolone $5 \mathrm{mg}$ after the meal.

8.002 tabl. / $2 / 2$

11.001 tabl. / 1/1

14.001 tabl. / $1 / 2$ / 0

3 days / 3 days / 3 days

Then dose reduction to 0

2) Furosemid $40 \mathrm{mg} \mathrm{1/2}$ tablet every day

3) Panangin $₫$ (magnesium asparagine $140 \mathrm{mg}$, potassium asparaginate $158 \mathrm{mg}$ ) 1 tabl. X 2 times a day after the meal

4) complete blood count, clinical chemistry: ALT, AST, total bilirubin, direct, $A F$, gamma-GT, total protein, creatinine, urea.

\subsubsection{7}

The skin process showed a positive dynamic: infiltration reduction and no itching. The patient received 2,5 tablets of steroids. Continuation of treatment. In clinical chemistry, there was a slight increase in direct bilirubin ( $5.9 \mu \mathrm{mol} / \mathrm{L}$ at a standard value of $5.0 \mu \mathrm{mol} /$ L), ALT (43.2 U / L at a normal value to $41 \mathrm{U} / \mathrm{L}$ ), a slight Lymphocyte increase $(3.56 \mathrm{Gpt} / \mathrm{L}$ at a standard value to 3.26 Gpt / L).

25.11. 2017

Laboratory data yielded a normalization of lymphocytes and continuous improvement in the skin process.

In addition to the treatment:

Salicylic acid 2.0

Sulfur 3.0

Birch wood tar 5,0

Zinc paste 100.0

Prednisolone $5 \mathrm{mg}$ after the meal,

8.002 tabl.

11.00 1/2 Panangin 1 tabl. X 2 times a day, after dinner Local treatment: equal + sulfur salicyl ointment. 


\subsubsection{7}

The laboratory values showed lymphocytes of $3.34 \mathrm{Gpt}$ / L (reduction), bilirubin directly of $6.0 \mu \mathrm{mol} / \mathrm{L}$. The skin process showed a steady clinical improvement.

Prescription ointment with birch wood tar at times, in the morning. Zinc oil x 2-3 times a day. $2 \%$ boric acid as a water layer.

In addition to treatment: Heptral ${ }$ (Ademetionin, Hepatoprotector, Abbot Laboratories, USA) 500 mg of Ion Ademetionin; 1 caps. $\mathrm{x} 2$ times a day, after meals.

\subsubsection{8}

It showed a steady improvement of the skin condition. The redness persisted in the areas where the ointment sulphur and zinc oxide was used. The ointment was prescribed and then discontinued due to severe aggravation in the first days of use.

\section{Prescription:}

1) Prednisolone 0.05 (1 tablet) per day

2) Furosemide $1 / 22$ times a week.

3) Heptral 1 tabl. 2 times a day.

4) Water pad with boric acid

5) Zinc oil

6) Local on the skin under the eyes - hydrocortisone eye ointment 2 times a day.

Reminder in 7 days.

\subsubsection{8}

In the skin process, the new papules on the chin skin developed (the patient worked in the frost, in colder and windy conditions). In addition to the treatment:

1) Prednisolone $10 \mathrm{mg}$ per os

2) Furosemide 1/2 2 times a week.

3) Hydrocortisone ointment on the nasal membrane once a day.

\subsubsection{8}

There was an improvement in the condition of the main lesion on the skin. Papular rashes remained on the skin under the lower eyelids. On the 22nd of January, 2018, the prescription of the synthetic retinoid Isotretinoin $20 \mathrm{mg}$ once daily was recommended when taking the hepatoprotector Adamethionin.

\subsubsection{8}

The continuation of the treatment in the abovementioned dose. Instead of Adamethionin, $250 \mathrm{mg}$ ursodeoxycholic acid with a hepatoprotective, choleretic, anticholestatic, hypocholesterolemic and immunomodulatory effects was prescribed . The patient continued receiving $5 \mathrm{mg}$ prednisolone in tablets.

\subsubsection{8}

The resorption of papules continued. Prednisolone 5 mg was discontinued, locally "Cetaphilß" as a cream and foam for facial washing was prescribed. The patient continued to take the synthetic retinoid Isotretinoin. The laboratory values were checked regularly every 2 weeks.

\subsubsection{8}

End of the 2nd month synthetic retinoid therapy. Additionally: a sun cream with UVF 50. Before sleeping zinc ointment $10 \%$ (DE).

\subsubsection{8}

Middle of the 3rd month synthetic retinoid therapy. The same dosage. Continuous clinical improvement. No new papules, no complaints by the patient. Reminder in 1 month.

\subsubsection{8}

End of the 3rd Isotretinoin therapy course. Control of clinical chemistry laboratory values. Daily UV protection 21.04.2018 Control of laboratory values.

11.08.2018 in May - July 2018 weekly meetings with correction of the dose of synthetic retinoid Isotretinoin at a dose of $10 \mathrm{mg}$ per day until complete deposition under the control of clinical chemistry parameters.

During a holiday in Turkey, despite the intensified protective measures (UV protection, etc.), an acute aggravation of the skin process (formation of new papular elements in the nasolabial angle range) and itching when bathing in the seawater occurred. A synthetic retinoid Isotretinoin was prescribed $10 \mathrm{mg}$ once a day.

\subsubsection{8}

With a suspicion of a mild form of Bourneville-Pringle disease (tuberous sclerosis), the patient was referred to the republican clinical hospital of the Ministry of Health of the Republic of Tatarstan for genetic counseling. The disease was not diagnosed.

Limitations of the study As a limitation, it is understood that we reported a single case at the institution.

Limitations of the study: As a limitation, it is understood that we reported a single case at the institution. 


\section{Discussion}

\section{Perioral Dermatitis}

Perioral dermatitis is an inflammatory disease of the skin of the face near the lips and wings of the nose. This is quite a common inflammatory skin disease that affects predominantly women [1]. The disease most often occurs in late adolescence, but can also occur in children in early childhood. Previously, the disease was described as a rash in young female adults [2]. Mokos et al. describes perioral dermatitis as a disease that primarily affects women from 15 to 45 years old as well as prepubescent boys [3].

Aetiology and Pathogenesis: The aetiology of perioral dermatitis has not yet been elucidated. However, there are many risk factors leading to this dermatosis. These include: disturbances in the function of the gastrointestinal tract, malfunctions in the endocrine system, hormonal imbalance, nervous disorders; predisposition of the facial skin to allergic reactions and the use of toothpastes containing fluoride $[4,5]$. The connection between the appearance of perioral dermatitis and the prolonged use of local glucocorticoids has been established [6].

The Clinical Picture: The skin pathological process is represented by grouped papulopustules from flesh to red coloured located on an erythematous background in the area around the mouth. Also, the periorbital and perinasal regions may be involved in the skin condiiton [7 With multiple rashes, peeling of the skin may be noted [8] According to Wollenberg, Bieber et al., PD occurs in 39\% perioral cases, in $13 \%$ perinasal and in $14 \%$ affecting both regions of the face [9].

Subjectively, a burning sensation, tightness of the skin, itching can be noted.

Treatment of perioral dermatitis is a complex and lengthy process. As a rule, at the first stage it should be advised to abandon the use of cosmetics, detergents, toothpaste containing fluoride and ointments containing glucocorticoids. Antihistaminic drugs could be prescribed in cases of itching.

\section{Atopic Dermatitis}

Atopic dermatitis (AD) is a type of inflammation of the skin that results in itchy, swollen and cracked skin, disruption of the skin barrier, and upregulation of type 2mediated immune responses [10]. The cause of AD remains unknown but it is assumed that various genetic, environmental and immunologic factors affect the mechanism of the disease [11]. Many people with AD have a positive family history in atopy that is often accompanied by asthma, allergic rhinoconjunctivitis likely because of shared immunogenetic and environmental mechanism [12]. AD is diagnosed clinically while various clinical criteria were developed. One of the best validated and frequently used works was presented by Hanifin and Rajka, named "UK diagnostic criteria"

\section{UK diagnostic Criteria}

An itchy skin condition (or parental report of scratching or rubbing in a child) plus 3 or more of the following:

1. History of involvement of the skin creases such as folds of elbows, behind the knees, fronts of ankles or around the neck (including cheeks in children under 10).

2. A personal history of asthma or hay fever (or history of atopic disease in a first-degree relative in children under 4).

3. A history of a general dry skin in the last year.4 Visible flexural eczema (or eczema involving the cheeks/forehead and outer limbs in children under 4).

4. Onset under the age of 2 (not used if child is under 4) [13].

Topical corticosteroids, such as hydrocortisone, have proven effective drugs in managing $\mathrm{AD}$. If topical corticosteroids are ineffective, short-term treatment with topical calcineurin inhibitors like tacrolimus or pimecrolimus may be tried [14]. Nevertheless, the use of the last is controversial and is associated with higher risk of skin cancer and lymphoma [14]. A systematic review conducted by Ashcroft, Chen et al. revealed that corticosteroids and tacrolimus are more effective than pimecrolimus [15].

\section{Acne Vulgaris}

The first morphological changes in acne are associated with the formation of retention hyperkeratosis of the follicle. Hyperplasia and hypersecretion of the sebaceous glands can be caused either by an increase in the level of sex hormones (absolute hyperandrogenemia) or an increase in the activity of type $5 \alpha$-reductase and / or an increase in the density of nuclear receptors for dihydrotestosterone (relative hyperandrogenemia) [16]. Acne could also have a microbial aetiology [17]. Jović, et al. assumes that psychological stress may also affect skin causing acne [18].

Retention hyperkeratosis and excessive sebum production lead to the formation of the primary element of acne - comedone. It obstructs the excretory duct of the sebaceous gland of the hair follicle [19]. The anaerobic 
conditions created as a result of this are optimal for the rapid growth and reproduction of $P$. acnes. The activity of P. acnes is associated with the formation of inflammatory elements of acne. As a result of chronic persistent inflammation, persistent dyschromia and scars form on the site of the resolved elements of acne [20].

Conflicts of interest statement: The authors declare that there are no conflicts of interest.

Acknowledgements: We would like to say many thanks to Mr. Timothy Peter DeCoursey for grammar and spelling check of our case study.

\section{References}

1. Lipozenčić J, Hadžavdić SL (2014) Perioral dermatitis. Clin Dermatol 32(1): 125-130.

2. Tolaymat L, Hall MR (2019) Dermatitis, Perioral. Treasure Island (FL), StatPearls.

3. Mokos ZB, Kummer A, Mosler EL, Čeović R, BastaJuzbašić A (2015) Perioral dermatitis: till a therapeutic challenge. Acta Clin Croat 54(2): 179-185.

4. Lee GL, Zirwas MJ (2015) Granulomatous Rosacea and Periorificial Dermatitis: Controversies and Review of Management and Treatment. Dermatol Clin 33(3): 447-455.

5. Peters P, Drummond C (2013) Perioral dermatitis from high fluoride dentifrice: a case report and review of literature. Aust Dent J 58(3): 371-372.

6. Sharma R, Abrol S, Wani M (2017) Misuse of topical corticosteroids on facial skin. A study of 200 patients. J Dermatol Case Rep 11(1): 5-8.

7. Kellen R, Silverberg NB (2017) Pediatric periorificial dermatitis. Cutis 100(6): 385-388.

8. Tilton EE, Bavola C, Helms SE (2015) Rash around the mouth: What is it? J Am Dent Assoc 146(5): 337-340.

9. Wollenberg A, Bieber T, Dirschka T, Luger T, Meurer M, et al. (2011) Periorale Dermatitis. JDDG: Journal der Deutschen Dermatologischen Gesellschaft 9(5): 422-428.

10. Cork MJ, Danby SG, Ogg GS (2019) Atopic dermatitis epidemiology and unmet need in the United Kingdom. J Dermatolog Treat 21: 1-9.

11. Grey K, Maguiness S (2016) Atopic Dermatitis: Update for Pediatricians. Pediatr Ann 45(8): e280-286.

12. Mathiesen SM, Thomsen SF (2019) The prevalence of atopic dermatitis in adults: systematic review on population studies. Dermatol Online J 25(8).

13. Williams HC, Burney PG, Pembroke AC, Hay RJ (1994) The U.K. Working Party's Diagnostic Criteria for Atopic Dermatitis. III. Independent hospital validation. Br J Dermatol 131(3): 406-416.

14. Berke R, Singh A, Guralnick M (2012) Atopic dermatitis: an overview. Am Fam Physician 86(1): 3542.

15. Ashcroft DM, Chen L-C, Garside R, Stein K, Williams HC (2007) Topical pimecrolimus for eczema. Cochrane Database Syst Rev (4): CD005500.

16. Bergler-Czop B (2014) The aetiopathogenesis of acne vulgaris - what's new? Int J Cosmet Sci 36(3): 187194.

17. Shaheen B, Gonzalez M (2011) A microbial aetiology of acne: what is the evidence? Br J Dermatol 165(3): 474-485.

18. Jović A, Marinović B, Kostović K, Čeović R, BastaJuzbašić A, et al. (2017) The Impact of Pyschological Stress on Acne. Acta Dermatovenerol Croat 25(2): 1133-1141.

19. Fox L, Csongradi C, Aucamp M, du Plessis J, Gerber M (2016) Treatment Modalities for Acne. Molecules 21(8): E1063.

20. Saurat JH (2015) Strategic Targets in Acne: The Comedone Switch in Question. Dermatology 231(2): 105-111. 